\title{
Original
}

\section{La web Familia y Salud como fuente de promoción de la salud de niños, familias y adolescentes}

\author{
M. E. Vázquez Fernández ${ }^{\mathrm{a}}$, J. J. Morell Bernabéb , J. J. Cuervo Valdés', E. Fernández Segurad, \\ F. J. Garrido Torrecillass, R. López García ${ }^{f}$ A. M. Lorente García Mauriñog, M. E. Serrano Poveda ${ }^{\text {h }}$
}

Publicado en Internet:

8-mayo-2015

Marta Esther Vázquez Fernández: mvmarvazfer@gmail.com

aPediatra. CS Arturo Eyries. Valladolid. España. Grupo Educación para la Salud de la AEPap. Equipo editorial de la web Familia y Salud • bPediatra. CS Zona Centro. Badajoz. España. Coordinador de la web Familia y Salud • CPediatra. CS Ciudad Jardín. Badajoz. España • dCS de Nerja. Málaga. España - ePediatra. EBAP UCG Churriana de la Vega (distrito metropolitano). Granada. España • fPediatra. CS Alhaurín el Grande. Málaga. España • EPediatra. CS San Juan de la Cierva. Getafe. Madrid. España - hPediatra. CS Benicalap Azucena. Valencia. España.

Introducción: las tecnologías de la información y comunicación se han convertido en un recurso donde el paciente busca información sobre salud y puede compartir experiencias.

Objetivos: analizar el flujo de comunicación generado por la web Familia y Salud desde su inicio en abril del 2012.

Metodología: se analiza el programa estadístico Google Analytics agregado a la web: número de vistas, usuarios, tiempo de duración de la sesión, porcentaje de rebote, perfil sociodemográfico, artículos más visitados y satisfacción.

Palabras clave:

- Web

- Internet

- Promoción de la

salud

- Salud infanto-juvenil
Resultados: se observa un notable incremento del número de visitas, usuarios y seguimiento en el último año, con un elevado nivel de satisfacción. Se reciben visitas de un gran número de países hispano hablantes.

Conclusiones: la web Familia y Salud se está convirtiendo en una poderosa herramienta de Internet para trasmitir información fiable relacionada con la salud infanto-juvenil.

\section{Familia y Salud web as source of health promotion for children, families and teens}

Introduction: information and communication technologies have become a resource where the patient seeks health information and shares experiences.

Objective: to analyze the flow of communication generated by the web Familia y Salud from the beginning, in April 2012.

Methodology: we have analyzed Google Analytics, a statistical program added to the web: number of views, users, duration of the session, bounce rate, socio-demographic profile, most popular articles and satisfaction.

Key words:

- Web

- Internet

- Health promotion

- Child and adolescent health
Results: we observed a significant increase in the number of visits, users and followers in the last year, with a high level of satisfaction. We received many visits from Spanish speaking countries.

Conclusions: the web Familia y Salud is becoming a powerful Internet tool for transmiting reliable information related to child and adolescent health.

Cómo citar este artículo: Vázquez Fernández ME, Morell Bernabé JJ, Cuervo Valdés JJ, Fernández Segura E, Garrido Torrecilllas FJ, López García R, et al. La web Familia y Salud como fuente de promoción de la salud de niños, familias y adolescentes. Rev Pediatr Aten Primaria. 2015;17:e117-e124. 


\section{INTRODUCCIÓN}

Las tecnologías de la información y comunicación se han convertido en una potencial fuente de promoción de la salud, en particular en Pediatría ${ }^{1,2}$. Muchos son los formatos de Internet para compartir conocimiento, desde los más conocidas (correo electrónico, listas de distribución, páginas web, etc.) hasta los más novedosas (como el fenómeno blog y las redes sociales) y todo lo que queda por llegar. Esta nueva forma de utilizar la red se llama web 2.0 (o "web social") y es una realidad ${ }^{3}$.

Cada vez más personas buscan en Internet información sobre salud y demandan este tipo de aplicaciones para buscar información o relacionarse con otros pacientes, instituciones o profesionales ${ }^{4,5}$. También cada vez son más los profesionales sanitarios que empezamos a ver esta nueva modalidad como una herramienta de trabajo, para mejorar nuestra formación, investigación o la asistencia que ofrecemos a los pacientes ${ }^{6-8}$.

La web Familia y Salud contiene información actualizada periódicamente (textos, podcast, vídeos, enlaces, imágenes), fiable y con elevado rigor científico, destinado a familias, profesionales, cuidadores, niños y adolescentes. Aunque da la posibilidad de interactuar mediante comentarios y opiniones, no pretende provisión de servicios médicos ni sustituir la consulta presencial. Con el fin de optimizar su uso, se divide en varias secciones con información de estilo en cascada:

- Crecemos: pretende informar y acompañar en los distintos momentos de la crianza de nuestros hijos. Hasta el momento tiene incorporados temas para cada tramo de edad en relación a los hábitos de salud, el desarrollo, la alimentación, la actividad física y el juego.

- Vivimos sanos: agrupa artículos de temáticas como la lactancia materna, la alimentación, la higiene bucodental, el sueño, etc.

- Podemos prevenir: sobre aspectos preventivos.

- Vacunas: preguntas frecuentes, calendarios vacunales, vacunación internacional y vacunas específicas.
- Nos preocupa que: agrupa temas de interés general por orden alfabético.

- Enfermedades: clasifica la información según la parte del cuerpo afectada.

- Recursos: decálogos, documentos, vídeos, cuentos, aplicaciones, blogs, juegos, podcast y otros recursos.

- Salud Joven: trece secciones destinadas a los adolescentes y jóvenes: mi cuerpo, sexualidad, ocio y tiempo libre, abuso de sustancias, experiencias...

- Medicinas: información práctica y normas de uso de medicinas de uso habitual en niños.

También incluye otros servicios como un sistema de registro, que facilita a los usuarios el acceso a los contenidos que son de su interés y recibir de forma periódica un boletín informativo, y una amplia inmersión en las redes sociales (Twitter ${ }^{\circledR}$ y Facebook $^{\circledast}$ ), que facilita la divulgación diaria de las novedades.

\section{METODOLOGÍA}

Tipo de estudio: análisis transversal descriptivo del flujo de comunicación de la web Familia y Salud (www.familiaysalud.com).

Tiempo de estudio: desde el 23/04/2012, fecha de inicio de las primeras publicaciones en la web hasta el 15/01/2015, fecha de la revisión.

Material de estudio: artículos y noticias elaborados por más de 230 pediatras y otros profesionales.

Metodología de revisión: se han analizado los datos contenidos en el programa de estadísticas Google Analytics ${ }^{\circledR}$, que permite conocer cómo interactúan los usuarios con la web. Además se han evaluado los resultados de una encuesta de satisfacción, online y voluntaria, sobre los contenidos de la web.

Variables analizadas: número de visitas, número de usuarios, páginas vistas/sesión, proporción de usuarios nuevos y revisitadores, tiempo de duración media de la sesión, porcentaje de rebote, datos sociodemográficos, cuáles son los contenidos 
más populares, seguidores en redes sociales, indicadores de "me gusta" y nivel de satisfacción de usuarios.

\section{RESULTADOS}

\section{Número de visitas y usuarios}

El número total de páginas vistas ha estado en torno a las 6,5 millones de páginas, con más de cuatro millones de usuarios. En el último año hemos recibido algo más de 3,6 millones de vistas procedentes de 2,47 millones de usuarios. En el último mes hubo en torno a 10000 visitas diarias. Se observa un incremento de las visitas a lo largo de los tres años de funcionamiento de la web, que se ha hecho exponencial en el último año (más del 500\% de incremento de visitas) (Fig. 1). El número de páginas vistas por sesión ha sido de 1,35.

La relación de usuarios nuevos fue $86,7 \%$ frenta al $13,4 \%$ que son usuarios que ya han utilizado la web (Fig. 2). Esto supone que más de medio millón de personas repiten visitas.
Las visitas fueron más bajas los fines de semana (sábado y domingo) y mayores los días de diario. El momento de mayor flujo de visitas fue de 18:00 a 23:00 horas.

Tiempo de duración media de la sesión

La duración media de la sesión fue de 1,16 minutos. Se muestra una tendencia estable desde el inicio de la web.

\section{Porcentaje de rebote}

Clara tendencia descendente: con una media del $35,7 \%$, hemos descendido al $15 \%$ en el último año.

\section{Perfil sociodemográfico}

El $61 \%$ de la población mayor de 18 años que nos consulta tenía entre 18 y 34 años (Fig. 3). El 45,8\% fueron mujeres, el 53,1 varones ( $p<0,005)$.

El país del que provienen más visitas es España (35,8\%), seguido de México (17,3\%) y otros países hispanohablantes. En España destacaron Madrid $(9,2 \%)$ y Barcelona $(3,9 \%)$ y de fuera, la ciudad de México (4,0\%) (Tabla 1).

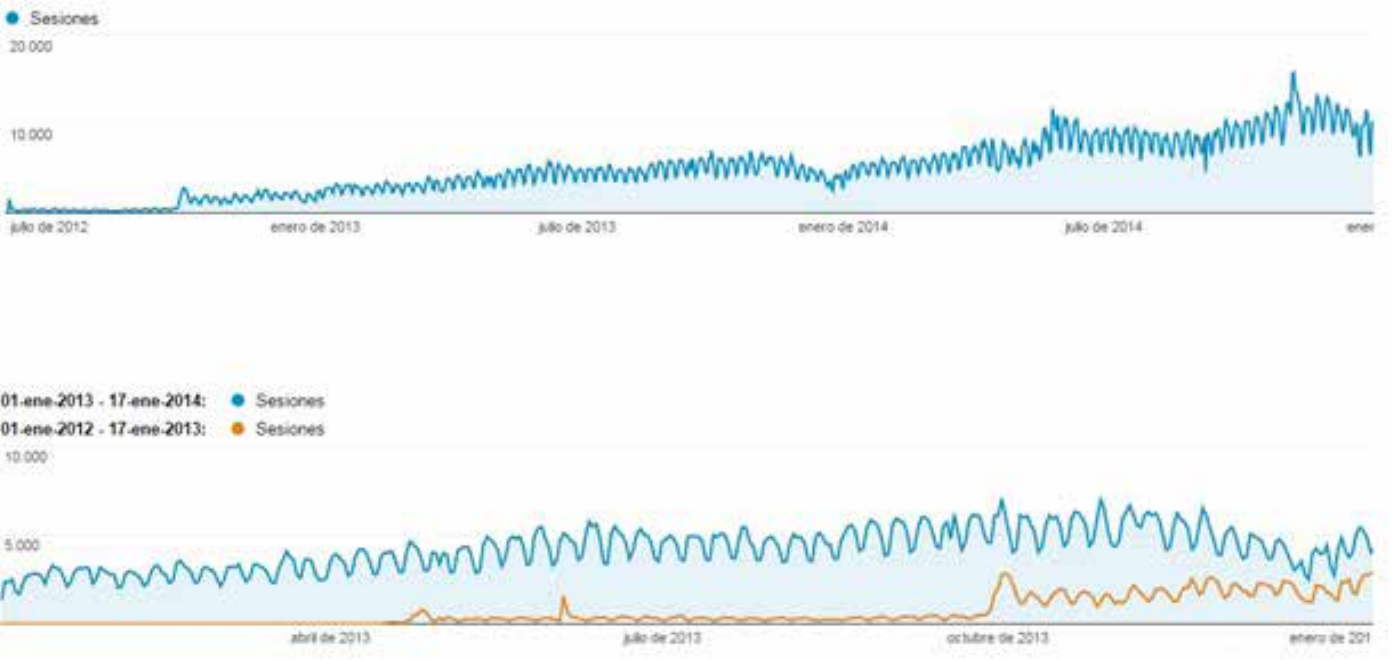




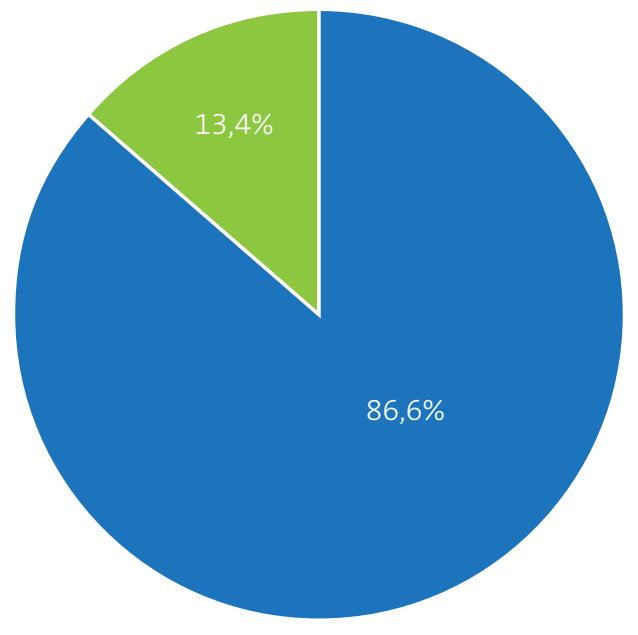

Nuevo visitante

Visitante repetidor

\section{Contenidos}

Los contenidos más visitados han sido la dislexia, los vómitos y las lombrices. Por secciones: Nos preocupa que (35,8\%), Enfermedades (19,6\%), Vivimos sanos, Crecemos y, en quinto lugar, Salud Joven (Tabla 2).

\section{Motor de búsqueda}

Los principales navegadores utilizados fueron Chrome (49,02\%), Android (18,17\%) y Safari (14,16\%) De manera global, el 50\% acceden a la web desde dispositivos móviles o tablets y, si nos referimos al último año, la cifra llega al 56,39\%.

\section{Figura 3. Visitas según la edad}

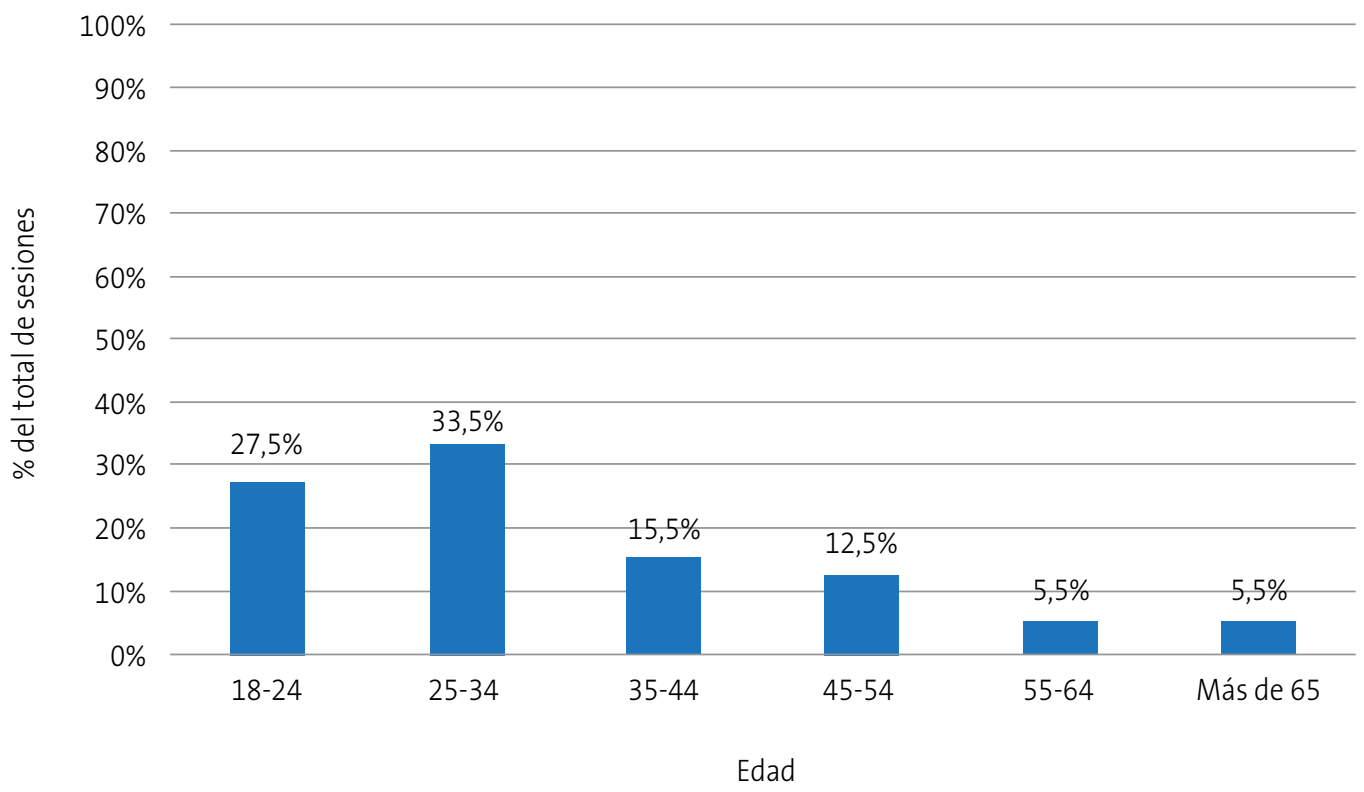




\begin{tabular}{|c|c|c|c|}
\hline \multicolumn{2}{|r|}{ País } & Sesiones & $\%$ sesiones \\
\hline 1 & España & 122690 & $35,78 \%$ \\
\hline 2 & México & 59360 & $17,31 \%$ \\
\hline 3 & Argentina & 26948 & 7,86 \\
\hline 4 & Chile & 20664 & $6,03 \%$ \\
\hline 5 & Colombia & 20230 & $5,90 \%$ \\
\hline 6 & Perú & 19865 & $5,79 \%$ \\
\hline 7 & EE. UU. & 17848 & $5,20 \%$ \\
\hline 8 & Venezuela & 13406 & $3,91 \%$ \\
\hline 9 & Ecuador & 9274 & $2,70 \%$ \\
\hline 10 & República Dominicana & 3378 & $0,99 \%$ \\
\hline
\end{tabular}

\section{Redes sociales}

En las dos redes sociales Twitter $^{\circledR}$ y Facebook $^{\circledR}$, el rendimiento ha sido progresivo desde su inicio. $\mathrm{Fa}$ milia y Salud tiene 1628 seguidores en Twitter ${ }^{\circledR}$, 1201 tuits y en Salud Joven 192 seguidores y 393 tuits. En Facebook ${ }^{\circledR}, 1500$ “Me gusta” y 229 “Me gusta" para Salud Joven.

\section{Encuesta de satisfacción}

En la encuesta de satisfacción online hemos obtenido 100 respuestas. De forma general, al 56\% de los encuestados la web le ha parecido muy útil, al $40 \%$ bastante útil, al $4 \%$ poco útil y a nadie nada útil. Por contenidos, la sección que más interés despierta es la de Enfermedades, seguida de Nos preocupa que y Salud Joven (Fig. 4).

\section{DISCUSIÓN}

Partimos de una realidad indiscutible: tres de cada cuatro personas se informan en Internet sobre salud. En nuestras consultas nos encontramos con un paciente experto y emponderado que busca información antes y después de visitarnos. El auge de Internet y las redes sociales ha sido espectacular en los últimos años ${ }^{9}$.

Si bien es verdad que en el momento actual son numerosas las fuentes de información relacionadas con atención a la salud infantil, existen pocos estudios que analicen su potencial de comunicación ${ }^{6,10}$. Todo ello hace que nuestros resultados sean difíciles de comparar con otros estudios.

El interés por la web Familia y Salud es alto, tenemos un gran número de visitas diarias (10 000 visitas al día), con una tendencia ascendente, tanto en el número de visitas como de nuevos visitantes

\begin{tabular}{|c|c|c|c|}
\hline \multicolumn{2}{|r|}{ Título de la página } & \multirow{2}{*}{$\begin{array}{c}\begin{array}{c}\text { Número } \\
\text { de páginas vistas }\end{array} \\
331805\end{array}$} & \multirow{2}{*}{$\begin{array}{c}\text { \% número } \\
\text { de páginas vistas } \\
5,42 \%\end{array}$} \\
\hline 1 & ¿Qué es la dislexia? & & \\
\hline 2 & ¿Qué hacer cuando mi hijo vomita? & 288099 & $4,71 \%$ \\
\hline 3 & Las lombrices, ¿cómo es posible que a mi hijo le hayan salido esos bichitos? & 218906 & $3,58 \%$ \\
\hline 4 & El recién nacido. Consejos para el primer mes de vida & 179438 & $2,93 \%$ \\
\hline 5 & Hongos en los pies, ¿qué son y qué podemos hacer? & 178498 & $2,92 \%$ \\
\hline 6 & Padres y pediatras al cuidado de la infancia y la adolescencia & 177708 & $2,90 \%$ \\
\hline 7 & Ganglios. Adenopatías: importancia y consencuencias & 132877 & $2,17 \%$ \\
\hline 8 & Cómo manejar la tos: qué hacer cuando mi hijo tose & 113666 & $1,86 \%$ \\
\hline 9 & Displasia de cadera & 113337 & $1,85 \%$ \\
\hline 10 & Mononucleosis infecciosa o enfermedad del beso & 105203 & $1,72 \%$ \\
\hline
\end{tabular}


Figura 4. Contenidos que han despertado más interés entre los encuestados (cuestionario online). Valores del 0 (mínimo) al 9 (máximo)

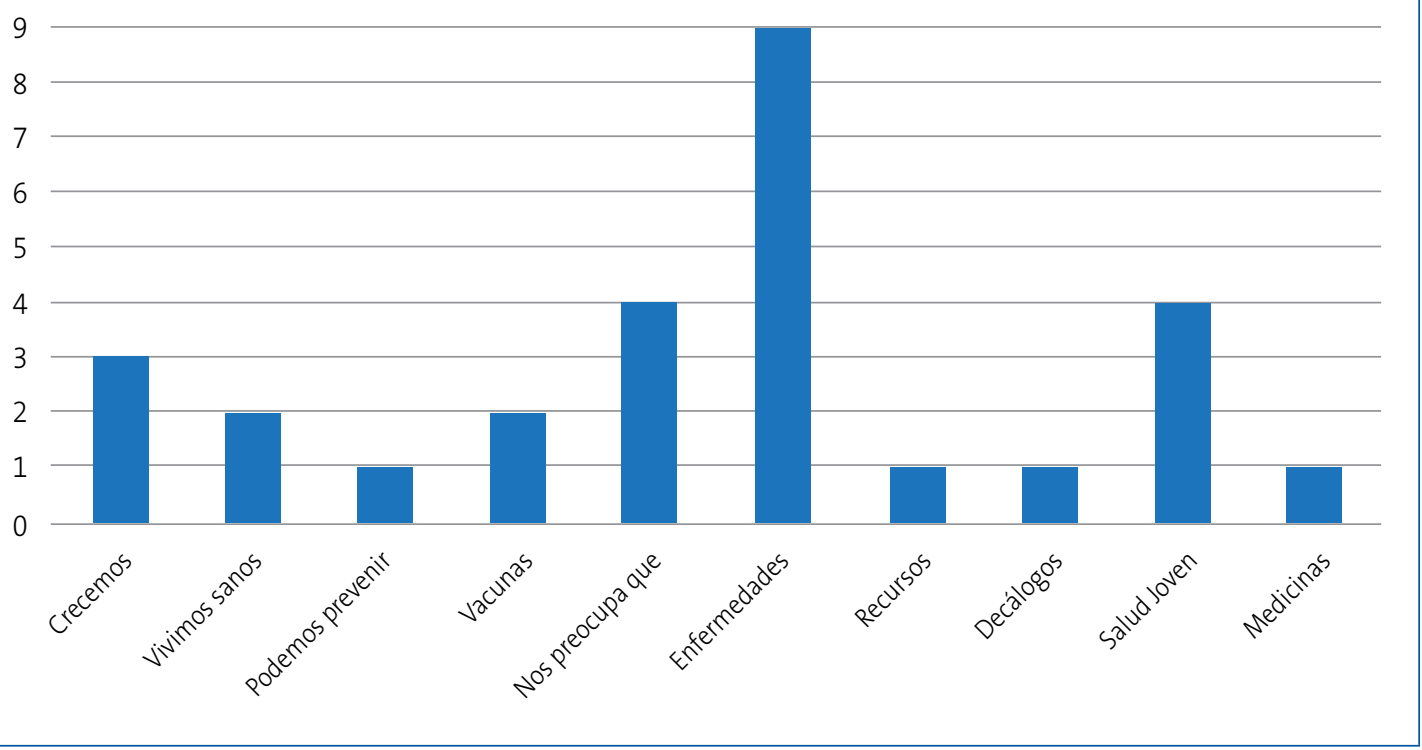

en los últimos meses. Cerca de medio millón de usuarios nos vuelven a visitar. Esto demuestra que la web gusta y despierta interés.

El tiempo de duración por sesión es otro indicador del interés que despierta una web. Se considera que cualquier web que supera el minuto está muy bien. Nuestra web supera este límite. La media mundial es de 20 segundos. Se supone que esto es porque hay miles de webs que realmente no dan respuesta a lo que el usuario busca, por lo que entra y abandona la página rápidamente.

El porcentaje de rebote es el ratio de visitas que entran en una página y no navegan más, salen sin leer más porque no les interesa. Cuanta más alta es la tasa de rebote menor calificación o ranking por parte de Google ${ }^{\circledR}$, lo que significa peor posicionamiento. En nuestro caso vamos consiguiendo mayor interés con un porcentaje de rebote cada vez más bajo.

Como no podía ser de otra manera para una web pediátrica dirigida a familias, el perfil de usuarios son población joven de 18 a 34 años, tanto hombres como mujeres, sin diferencias estadísticamente significativas. Además, a menor edad mayor uso de Internet. Algunos datos de trabajos publicados cifran que un $83 \%$ de los jóvenes utiliza los buscadores online para buscar información sobre salud ${ }^{3}$. Este dato es superior a las visitas a la consulta del médico (77\%) o las consultas al farmacéutico (46\%). Aunque también refieren que la fiabilidad que le dan a la información de Internet es menor que a la consulta presencial. Esto viene dado por una parte por una mayor accesibilidad, comodidad y rapidez que ofrece el canal online por sí solo.

Respecto a las visitas que procedieron de fuera de España, destaca la enorme representación de las zonas de habla hispana, dos de cada tres visitantes, lo que convierte a Familia y Salud en una de las principales webs en español sobre salud infantil. Internet es la autopista de la globalización, las distancias desaparecen, la información fluye de forma instantánea, la difusión es ilimitada, el acceso es ubicuo.

Los contenidos que han despertado más interés son los problemas de salud, destacando la dislexia y los vómitos. Esto confirma que el motor de búsqueda de información sobre salud en Internet es la enfermedad o dolencia y menos veces los aspectos preventivos relacionados con la nutrición, vacunas, 
estilos de vida saludable y medicamentos. Estos datos coinciden con el estudio publicado por el Observatorio Nacional de las Telecomunicaciones y la Sociedad de la Información (ONTSI) y la Entidad Pública Empresarial Red.es ${ }^{9}$. Por otro lado, los temas relacionados con la salud del adolescente, reunidos en la sección de Salud Joven ocupan un quinto lugar, que no está nada mal teniendo en cuenta que es la franja de población menos numerosa y con menor número de requerimientos médicos en las consultas habituales.

Un gran porcentaje de los usuarios que usaron nuestra web utilizaron en primera instancia un buscador para acceder a la información demandada. El siguiente paso fue introducir el nombre de la enfermedad/dolencia. Los móviles son los dispositivos desde los cuales tres de cada cutro visitantes acceden a nuestra web, seguido del ordenador de sobremesa.

Hoy en día no podemos ignorar el papel que desempeñan las redes sociales ${ }^{11}$. En nuestra web aprovechamos el potencial que estas ofrecen. Twitter $^{\circledR}$, Facebook ${ }^{\circledR}$ y otras redes sociales se convierten cada día con más intensidad en un punto de encuentro para intercambiar información y recursos que se van incluyendo en la web. Están evolucionando rápidamente y la web cada vez cuenta con más interacción con el usuario a través de este medio.

\section{BIBLIOGRAFÍA}

1. Jovel AJ. El paciente del siglo XXI. An Sist Sanit Navar. 2006:29:85-90

2. González de Dios J, Aleixandre Benavent R. Formación e información en Pediatría: aproximación a la necesidad de los profesionales. Acta Pediatr Esp. 2010; 68:235-40

3. Cepeda, JM. Manual de inmersión 2.0 para profesio nales de salud. En: Salud Conectada [en línea] [consultado el 05/05/2015]. Disponible en http://salud conectada.com/
La satisfacción de este tipo de herramientas online sobre salud es muy elevada. En nuestro caso, el 96\% de los usuarios piensan que la web es muy útil o bastante útil, siendo la sección Enfermedades la más apreciada.

\section{CONCLUSIONES}

Las búsquedas de información por Internet se están convirtiendo en un canal donde el paciente busca información, un lenguaje claro y cercano y poder compartir experiencias. La aparición de estos nuevos modelos no implica que todos los pacientes vayan a utilizarlos, o que vayan a ser en el futuro totalmente empoderados y activos y vayan a asumir un alto grado de responsabilidad en la toma de decisiones. Es posible que sigan existiendo muchas personas que prefieran seguir derivando las decisiones sobre su salud a los profesionales. También habrá otras que sí adquirirán esa responsabilidad y necesitarán de nosotros otro tipo de tratamiento.

La web Familia y Salud está logrando ser un medio poderoso de comunicación y promoción de la salud de los niños y adolescentes, un megáfono para difundir conocimientos y crear cultura sanitaria.

4. López de Ayala López MC, Catalina García B, Alfageme Pardo P. Hábitos de búsqueda y confianza en la información online sobre salud entre jóvenes universitarios. Actas del VI Congreso Internacional Latina de Comunicación Social (VI CILCS), Universidad de La Laguna, diciembre de 2014. En: Revista Latina de Comunicación Social [en línea] [consultado el 05/05/2015]. Disponible en www.revistalatinacs. org/14SLCS/2014_actas/017_Lopez.pdf

5. El rol de internet en el proceso de consulta de Información sobre salud. Encuesta Pfizer y The Cocktail Analysis. Septiembre 2010. En: Pfizer [en línea] [consultado el 05/05/2015]. Disponible en 
www.pfizer.es/docs/pdf/noticias/Resultados_en cuesta_Pfizer.pdf

6. Álvarez Rodríguez C, Aramburu Vilariño FJ, Fandiño Orgueira JM, Vázquez Lima M, Díez Lindín O, Bugarín González R. Valoración de la eficacia de un blog de medicina de urgencias como medio de comunicación. Emergencias. 2007;19:180-6.

7. Nuño-Solinis R, Rodríguez-Pereira C, Piñera-Elorriaga K, Zaballa-González I, Bikandi-Irazabal J. Panorama de las iniciativas de educación para el autocuidado en España. Gac Sanit. 2013;27:332-7.

8. González de Dios J, González Muñoz M, González Rodríguez P, Esparza Olcina M J, Buñuel Álvarez JC. Blogs médicos como fuente de formación e información: el ejemplo del blog Pediatría basada en pruebas. Rev Pediatr Aten Primaria. 2013:15:27-35.
9. Observatorio Nacional de las Telecomunicaciones y Sociedad de la Información (ONTSI). Los ciudadanos ante la e-Sanidad. Estudio sobre opiniones y expectativas de los ciudadanos sobre el uso y aplicación de las TIC en el ámbito sanitario. En: ONTSI [en línea] [consultado el 05/05/2015]. Disponible en http:// www.ontsi.red.es/ontsi/sites/default/files/informe_ ciudadanos_esanidad.pdf

10. Marín-Torresa V, Valverde Aliaga J, Sánchez Miró I. Internet como fuente de información sobre salud en pacientes de atención primaria y su influencia en la relación médico paciente. Aten Primaria. 2013;45:46-53.

11. Traver Salcedo V, Fernandez-Luque L. El e-paciente y las redes sociales. En: Salud 2.0 [en línea] [consultado el 05/05/2015]. Disponible en www.salud20.es/ wp-pdf.php?f=178 